\title{
Auxiliary Stopping Area Layout Method for High-Speed Maglev Operated Bidirectionally on Single Track
}

\author{
Yi Yu $\mathbb{D}^{1},{ }^{1}$ Pengzi Chu $\mathbb{D}^{1,2}$ Danyang Dong, ${ }^{1}$ Xi Jiang, ${ }^{3}$ Huahua Zhao, ${ }^{1}$ and Hui Lin ${ }^{1}$ \\ ${ }^{1}$ Maglev Transportation Engineering R\&D Center, Tongji University, Shanghai 201804, China \\ ${ }^{2}$ The Key Laboratory of Road and Traffic Engineering, Ministry of Education, Tongji University, Shanghai 201804, China \\ ${ }^{3}$ China Railway Siyuan Survey and Design Group Co., Ltd., Wuhan 430063, China \\ Correspondence should be addressed to Pengzi Chu; cpz_myhk@163.com
}

Received 15 January 2021; Revised 27 March 2021; Accepted 9 April 2021; Published 22 April 2021

Academic Editor: Yuan Gao

Copyright (c) $2021 \mathrm{Yi} \mathrm{Yu} \mathrm{et} \mathrm{al.} \mathrm{This} \mathrm{is} \mathrm{an} \mathrm{open} \mathrm{access} \mathrm{article} \mathrm{distributed} \mathrm{under} \mathrm{the} \mathrm{Creative} \mathrm{Commons} \mathrm{Attribution} \mathrm{License,} \mathrm{which}$ permits unrestricted use, distribution, and reproduction in any medium, provided the original work is properly cited.

\begin{abstract}
Auxiliary stopping area (ASA) is the necessary emergency facility for train safety of the normal high-speed maglev. The study addresses the ASA layout problem of the high-speed maglev operated bidirectionally on single track. First, an optimization model of the ASA layout for unidirectional double-track lines considering train safety, operation efficiency, and construction cost is established, and two basic methods of the ASA layout are investigated based on the distance demand characteristic of ASAs. Then, the ASA layout problem of bidirectional single-track lines is analyzed, and an ASA two-way coordination layout algorithm (ASATWCLA) is proposed. Finally, a numerical experiment is carried out. The results suggest that under the premise of train safety and operation efficiency, compared with using the basic methods separately on the two directions, adopting the ASA-TWCLA algorithm can obtain a more economical ASA layout scheme for the same scenario.
\end{abstract}

\section{Introduction}

With the advantages of strong climbing ability, low noise, green, and so on, maglev transportation has attracted more and more attention [1-3]. Maglevs have already served the public in Beijing, Changsha, Incheon, Nagoya, and Shanghai. Many maglev lines are also under construction. Unlike the wheel-rail transportation system, the driving power of the maglev train comes from the track, and the train does not touch the track during operation [1-5]. As a result, the safety protection strategies of the maglev transportation system are different to the wheel-rail transportation system.

To ensure train safety, the operation control system (OCS) of maglevs needs to have a complete safety protection function. The 2-dimension (2D) speed protection mode, which not only prevents the train speed from exceeding the maximum speed profile but also prevents the speed from falling below the minimum speed profile, is one of the important safety contents for the normal high-speed maglev (i.e., the high-speed room temperature maglev train) $[1,6-10]$. The auxiliary stopping area (ASA) is the emergency facility equipped with power rails and evacuation exits in the interstation section. Its construction cost is high, but it is a basis for realizing the 2D speed protection mode. Based on the 2D speed protection mode and ASAs, the normal high-speed maglev (HSM) train can safely stop and start again when encountering emergencies $[1,6,7]$. The research on the layout of ASAs is beneficial to the engineering practice of the HSM.

1.1. Literature Review. Few studies have focused on the layout of ASAs. In terms of the unidirectional double-track line (unidirectional line, for short) for the middle-to-high speed maglev, Lai et al. [11] took the area of the safe speed region and the Euclidean distances between the switching point of the maximum speed limit profile and the intersections of safe braking profiles and safe levitation profiles as optimal objects, regarded the minimum length of the ASA and the total length of ASAs as constraints, and solved the problem by the genetic algorithm. For the line of the HSM, Bian [12] suggested the intervals between ASAs for different 
speed ranges. Yu et al. [6] suggested an ASA layout method based on the protection speed, including the approach to set up and adjust ASAs for multiple target speed profiles. The method obtains the safety levitation profile of the terminal station firstly and then obtains the safe braking profile of a new ASA according to the intersection of the safety levitation profile and the target speed profile (TSP). Afterwards, the terminal-end of the ASA can be determined according to the foothold of the safety braking profile, and the head-end of the ASA can be obtained according to the length of the ASA. Based on the new ASA, the process can be repeated until the layout is complete [6]. Based on the study of $\mathrm{Yu}$ et al. [6], Jiang et al. [13] further discussed an ASA layout method considering train tracking operation. The method improves the operation efficiency by setting up ASAs where the train tracking intervals can be reduced.

Unlike the wheel-rail transportation system, the layout of ASAs affects the train operation control strategy, especially the train speed trajectory (i.e., the train speed profile). For the wheel-rail transportation system, the research on the optimization of a train speed profile is relatively mature. Asnis et al. [14] suggested that an optimal train speed profile should be composed of four operating conditions, involving in maximum traction, cruise, coasting, and maximum braking. Yang et al. [15] discussed a train speed profile optimization method based on the heuristic genetic algorithm. Taking the coasting section and braking point into account, Cao et al. [16] used the random reinforcement genetic algorithm to optimize the train speed profile. By abstracting a speed-distance network, Wang et al. [17] established a stochastic constrained shortest path model to optimize the train speed profile. For the normal middlespeed maglev (MSM) or the normal high-speed maglev, the stopping point stepping operation mode restricts the safe speed region of trains. Once ASAs are fixed, the safe speed region available for train operation is also limited. Yang [18] discussed the phenomenon earlier and designed methods for solving the time-saving speed profile and the energy-saving speed profile. Chai et al. [19] regarded the location of ASAs as constraints and proposed a solution for the energy-saving speed profile by first analyzing the conversion points of train operating conditions and then carrying out multi-interval optimization. For the middle-to-high speed maglev, Lai et al. [20] took the location of ASAs as constraints and optimized the train speed profile by using dynamic programming with mutative spacing. Taking ASAs, the nonlinear resistance, and the suspension energy consumption into account, Lai et al. [21] further studied the problem of reducing the energy consumption of the MSM train based on the dynamic programming and the mixed-integer linear programming. Taking the temperature and states of the on-board battery into consideration, Yang et al. [22] suggested the operation strategy of the train in propulsion decentralizations to ensure that it can stop at ASAs in case of emergencies.

For the train tracking interval, it refers to the minimum time interval (i.e., train tracking interval time) or the minimum distance interval (i.e., train tracking interval distance) between the following train and the preceding train. The shorter the tracking interval time, the greater the train passing capacity and the operation efficiency for a line. Train tracking intervals are divided into four typical categories, one is the section tracking interval and the others are station tracking intervals, which involve the departure tracking interval, the arrival tracking interval, and the passing tracking interval $[23,24]$. The train tracking intervals of the wheel-rail transportation system are affected by train speed, signal equipment, signal block mode, and so on [25-27]. For the maglev transportation system, a maglev line is divided into some propulsion decentralizations, and each propulsion decentralization in the interstation section can only guide one train $[2,13,28,29]$. Based on this, the operation section of the maglev transportation system similar to the block section of the wheel-rail transportation system is also divided according to propulsion decentralizations. Since the HSM train runs according to the stopping point stepping mode and there can only be one train in a propulsion decentralization, the tracking intervals of maglev trains are affected by the length of propulsion decentralizations and the location of ASAs [13, 28, 29]. Ding et al. [29] studied the characteristics of the medium-speed maglev ASAs and discussed the calculation method of the section tracking interval. Jiang et al. [13] discussed calculation methods for the section tracking interval, the departure tracking interval, and the arrival tracking interval of the HSM and suggested that the layout of ASAs has an influence on the section tracking interval and the arrival tracking interval. Therefore, by setting ASAs at appropriate positions, the efficiency of train tracking operation can be improved.

In addition, for the $2 \mathrm{D}$ speed protection profiles (i.e., maximum speed profile and minimum speed profile), Chen and $\mathrm{Xu}$ [8] and Liu and $\mathrm{Xu}$ [9] introduced the calculation process of the two speed profiles for the normal high-speed maglev. When calculating the two speed profiles of the HSM, Zhao et al. [7] and Yang and Tang [10] further considered the most adverse condition to ensure train safety as far as possible.

1.2. Contribution and Organization. The layout of ASAs is related to train safety, operation efficiency, and construction cost $[12,13,21]$. However, the universality of previous methods is limited. In previous studies on the layout of ASAs, there is no consideration of line conditions. For example, ASAs should not be set up on bridges, switches, and steep gradients [12]. At the same time, the method suggested by Lai et al. [11] does not fully consider the 2D speed protection mode, which may cause the train to fail to operate according to the expected target speed profile. As a system engineering, train safety, line condition, economy, train speed profile, and tracking interval should be considered while setting up ASAs.

Moreover, enlightened by the operation of the Shanghai Maglev Demonstration Line and wheel-rail transportation systems, the research on the ASA layout problem of the normal high-speed maglev should consider the bidirectional operation on a single track. There are two reasons for this. First, when one track of a double-track line fails, the train can be operated on the opposite track to keep the 
normal operation as far as possible. For example, Xu et al. [30] discussed how to adjust train operation with the help of the opposite track when one track fails. For a doubletrack line of the HSM, if the bidirectional operation is considered when setting up ASAs, the robustness of its operation can be improved. Meanwhile, there are many single-track lines in the wheel-rail transportation system. The HSM has the advantage of high speed. If the station spacing and the passenger volume of an HSM line is suitable, a single track can also satisfy the needs of normal operation. There may be a demand for the bidirectional single-track line (bidirectional line, for short) of the HSM in the future.

However, to the best of our knowledge, the ASA layout problem considering train safety, bidirectional operation, line condition, economy, train speed profile, and tracking interval simultaneously has not yet been discussed. Compared with the previous studies, this paper has two main contributions: (1) the ASA layout methods considering line conditions for unidirectional lines and (2) the ASA layout method for bidirectional lines.

The rest of the study is as follows. The train operation process of the HSM and two ASA layout methods for unidirectional lines are investigated in Section 2. In Section 3 , the ASA layout problem for bidirectional lines is further reported, and a method named ASA two-way coordination layout algorithm (ASA-TWCLA) is proposed. The results of the numerical experiment are discussed in Section 4. Section 5 is the conclusion.

\section{Research Fundamentals}

2.1. Train Operation of the HSM. The operation control system (OCS) of the HSM realizes operation safety protection by monitoring the train speed, the maximum speed limit profile, and the 2-D speed protection profiles. As shown in Figure 1, to ensure that the train can stop at ASAs, when the train speed crosses the maximum speed profile, OCS will take actions to prevent the train speed profile from touching the safe braking profile and entering the dangerous speed region. When the train speed crosses the minimum speed profile, OCS will also take actions to prevent the train speed profile from touching the safe levitation profile and entering the dangerous speed region [7, 30]. Meanwhile, the OCS uses the stopping point stepping mode to ensure the ability of HSM trains to stop at ASAs. Specifically, in the operation process, the train runs forward with taking the current ASA as the target stopping point. Only when the train speed exceeds the minimum speed profile of the next stopping point (i.e., next ASA) and below the maximum speed profile of the current stopping point (i.e., current ASA), the train can take the next ASA as the target stopping point and runs forward.

The operation process of an HSM train can be described according to Figure 2. When the train departs from Station $O$, the end point of the accelerating zone is regarded as the current stopping point. Meanwhile, the OCS monitors the train speed profile and the corresponding protection profiles $[7,31]$. After the train speed is near the point $\operatorname{IDP}_{O, 1}$ (i.e., the

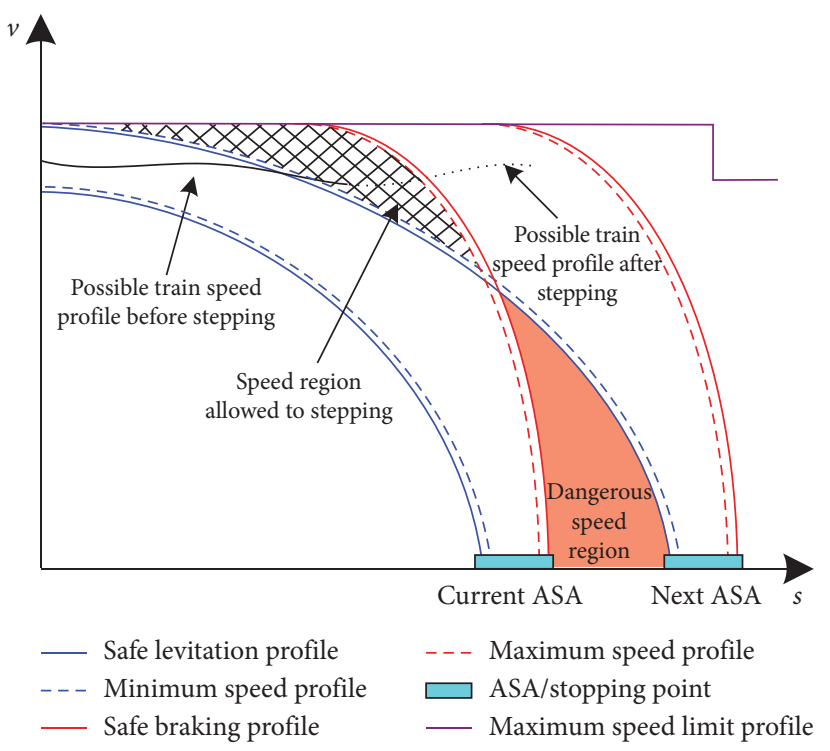

FIgURE 1: Stopping point stepping mode of the HSM.

intersection of the maximum speed profile of Station $O$ and the minimum speed profile of ASA 1) and meets the stopping point stepping requirement, the stopping point stepping is executed. Then, ASA 1 is taken as the current stopping point. By analogy, when the train completes the stopping point stepping near the point $\mathrm{IDP}_{4, D}$, it can run to Station $D$.

2.2. Basic Model of ASA Layout. The layout of ASAs in the study is to make the number of ASAs between stations as small as possible while ensuring train safety and operation efficiency. As shown in Figure 3, if the section $O D$ is divided into subsections from point $O$ to point $D$, to minimize the number of cut points, we can maximize the length of each subsection. At the same time, if the distance between point 1 and point $O$ is the maximum (i.e., $L_{O, 1}$ ) and the mileage between point 2 and point 1 is the maximum (i.e., $L_{1,2}$ ), then the mileage between point $\mathrm{O}$ and point 2 reaches the maximum (i.e., $L_{O, 2}$ ). In other words, the number of ASAs as small as possible is equivalent to the distance between an ASA and the origin station (or the terminal station) as large as possible.

For modeling, we first assume that the track sections which are unsuitable for setting up ASA are all short. That is, it will not cause the phenomenon that the train could not step successfully even if ASAs are set at the both ends of the section. Then, an ASA set $I$ is defined, and its index is $i$. $\mathrm{RP}_{i}$ is the head-end of ASA $i$, which is defined as reachable point (RP), and $\mathrm{HP}_{i}$ is the terminal-end of ASA $i$, namely, hazard point (HP) $[7,31]$, as shown in Figure 2 . Reachable points are the intersections of safe levitation profiles and the mileage axis, and hazard points are the intersections of safe braking profiles and the mileage axis. According to the criterion that the distance between an ASA and the origin station (or the terminal station) as large as possible, the objective function in the study can be expressed as follows: 


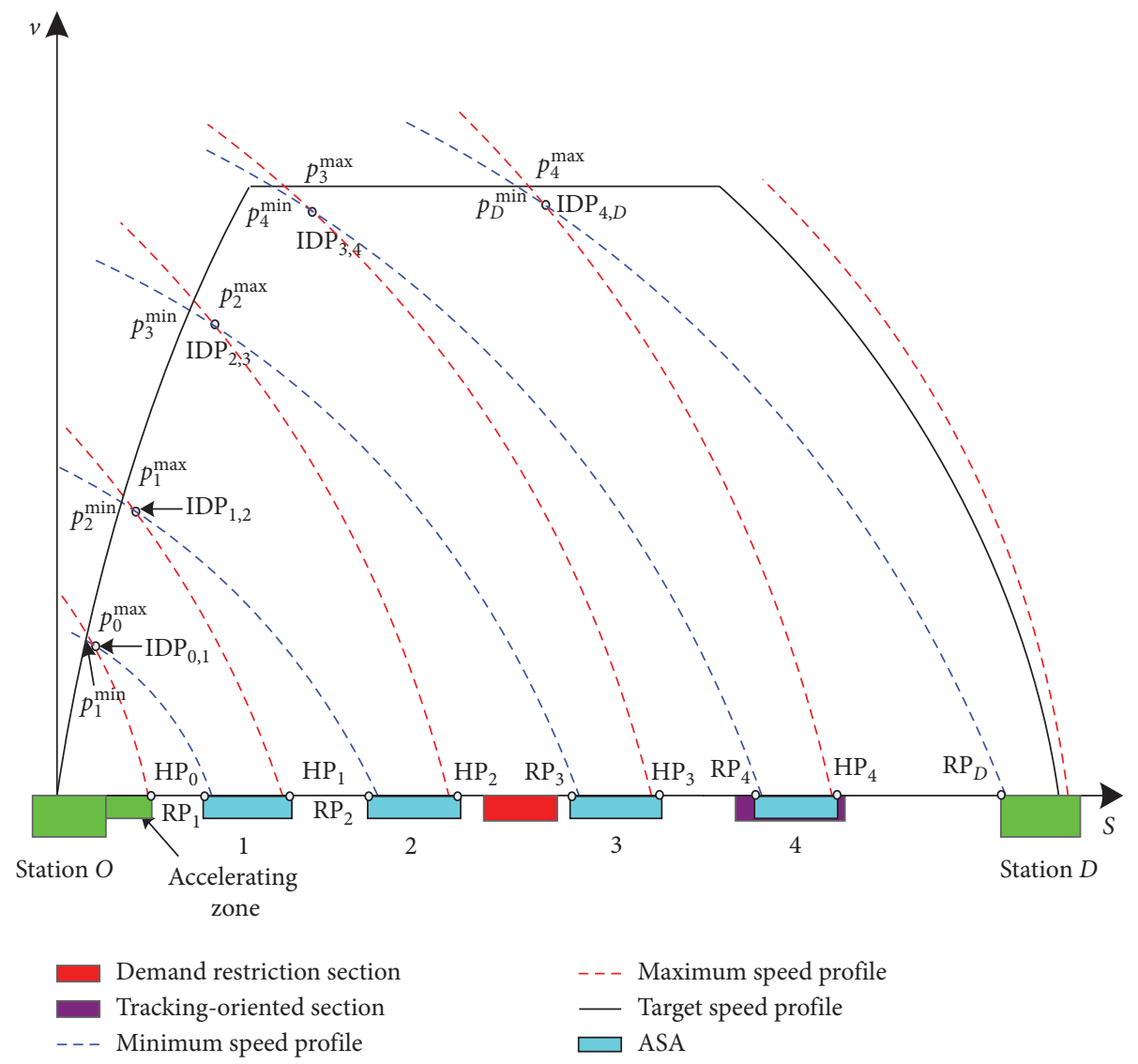

Figure 2: Train operation process of the HSM.

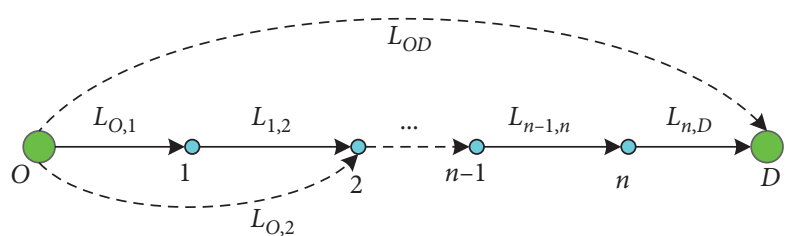

Figure 3: Analysis for the fewest cut points.

$$
\max \left(\mathrm{RP}_{i}-\mathrm{HP}_{O}\right), \quad \forall i \in I,
$$

or

$$
\max \left(\mathrm{RP}_{D}-\mathrm{HP}_{i}\right), \quad \forall i \in I \text {. }
$$

In Figure 2, point $p_{i}^{\min }$ is the intersection of the minimum speed profile from ASA $i$ and the target speed profile. Point $p_{i}^{\max }$ is the intersection of the maximum speed profile from ASA $i$ and the target speed profile. Meanwhile, we define the time interval between the point $p_{i+1}^{\min }$ and the point $p_{i}^{\max }$ as the stepping window time $\mathrm{SWT}_{i, i+1}$. According to the stopping point stepping operation mode, the train needs to complete the stopping point stepping from stopping point $i$ to stopping point $i+1$ within the time interval $\mathrm{SWT}_{i, i+1}$. Since the process of a stopping point stepping takes time, the stepping window time should be greater than 0 . In this regard, we define a stepping redundancy time SRT to restrict the stepping window time [6]. That is, stepping redundancy time is a time window considering enough redundancy, and a normal stepping process can be completed during the time window. When designing an ASA layout scheme, each stepping window time should not be less than SRT. Thus, there are three constraints for the model as follows:

$\mathrm{SWT}_{i, i+1} \geq \mathrm{SRT}, \quad \forall i \in I$,

$$
\mathrm{SWT}_{O, 1} \geq \mathrm{SRT}
$$

$$
\mathrm{SWT}_{\max (I), D} \geq \mathrm{SRT} .
$$

According to the attributes of track sections, some track sections should be considered setting up ASAs to reduce train tracking intervals $[13,29]$, and this type of section is defined as the tracking-oriented section (TOS) in the study, as shown in Figure 2. Moreover, if a certain track section is not suitable for setting up ASAs, the section is defined as the demand restriction section (DRS) in the study, as shown in Figure 2. For example, ASAs should not be set at bridges, switches, steep gradients, and so on [12]. For the line conditions considered above, a set TOS containing $\mathrm{M}$ tracking-oriented sections is defined, and its index is $m$. At the same time, a piecewise function $f_{\text {tos }}(I, m)$ is defined. If there are ASAs located at a tracking-oriented section tos $m$, $f_{\text {tos }}(I, m)$ is equal to 1 ; otherwise, it is equal to 0 . Then, there is a constraint for tracking-oriented sections as follows: 


$$
\sum_{m=1}^{M} f_{\mathrm{tos}}(I, m)=M, \quad \forall m \in\{1,2,3, \ldots, M\} .
$$

Moreover, we define a set DRS containing $\mathrm{N}$ demand restriction sections, and its index is $n$. Simultaneously, a piecewise function $f_{\mathrm{drs}}(i, n)$ is defined. If ASA $i$ is located at demand restriction section $\operatorname{drs}_{n}, f_{\text {drs }}(i, n)$ is equal to 1 ; otherwise, it is equal to 0 . Then, a constraint is acquired for demand restriction sections as follows:

$$
f_{\mathrm{drs}}(i, n)=0, \quad \forall i \in I, \forall n \in\{1,2,3, \ldots, N\} .
$$

In addition, ASAs should be located at the target line. And there are two constraints for the requirement as follows:

$$
\begin{aligned}
& \mathrm{HP}_{i}>\mathrm{HP}_{O}, \quad \forall i \in I, \\
& \mathrm{HP}_{i}<\mathrm{RP}_{D}, \quad \forall i \in I .
\end{aligned}
$$

\subsection{Basic Solution of ASA Layout for Unidirectional Line.} The decision variable of the ASA layout model (i.e., Equations (1)-(9)) is the positions of ASAs, but the total number of ASAs is unknown. The length of a line also makes the solution space very large. At the same time, the model is nonlinear, and some constraints need to be judged by simulation (e.g., Equations (3)-(5)). To find an optimal solution efficiently, the corresponding algorithm needs to be designed, especially the algorithm that considers the demand characteristics of ASAs. In this regard, based on the analyses of the characteristics, corresponding strategies are designed, and two heuristic algorithms for unidirectional lines are proposed.

First, Figure 4 shows the distance demand between two adjacent ASAs. The target speed profile first intersects the minimum speed profile of ASA $i+1$ and then intersects the maximum speed profile of ASA $i$, which meets the stopping point stepping requirement, and the time interval $\mathrm{SWT}_{i, i+1}$ between $p_{i+1}^{\min }$ and $p_{i}^{\max }$ is large. According to the objective function (i.e., Equation (1)) and the constraints (i.e., Equations (3)-(5)), when the time interval is exactly equal to the stepping redundancy time $S R T$, the distance between ASA $i$ and ASA $i+1$ is maximum $[6,13]$. And for the stopping point stepping requirement, the section between ASA $i$ and ASA $i+1$ is the feasible region at this moment. Therefore, if the position of ASA $i$ is fixed, the ASA can be regarded as a reference (i.e., reference ASA), and the position of ASA $i+1$ with the maximum distance from ASA $i$ can be determined at the right end of the feasible region based on the stepping redundancy time SRT, the reference ASA, and the target speed profile. As shown in Figure 4, ASA $i+1^{\prime}$ is the expected ASA.

For tracking-oriented sections, as shown in Figure 5(a), ASA $i+1$ is obtained based on ASA $i$ and the stepping redundancy time, but there is a tracking-oriented section between them. If we ignore the section, the result violates the constraint of Equation (6). According to the objective function that ASAs should be as far as possible from the origin station (Equation (1)), ASA $i+1$ should be set at the right margin of the tracking-oriented section, as shown in Figure 5(b).

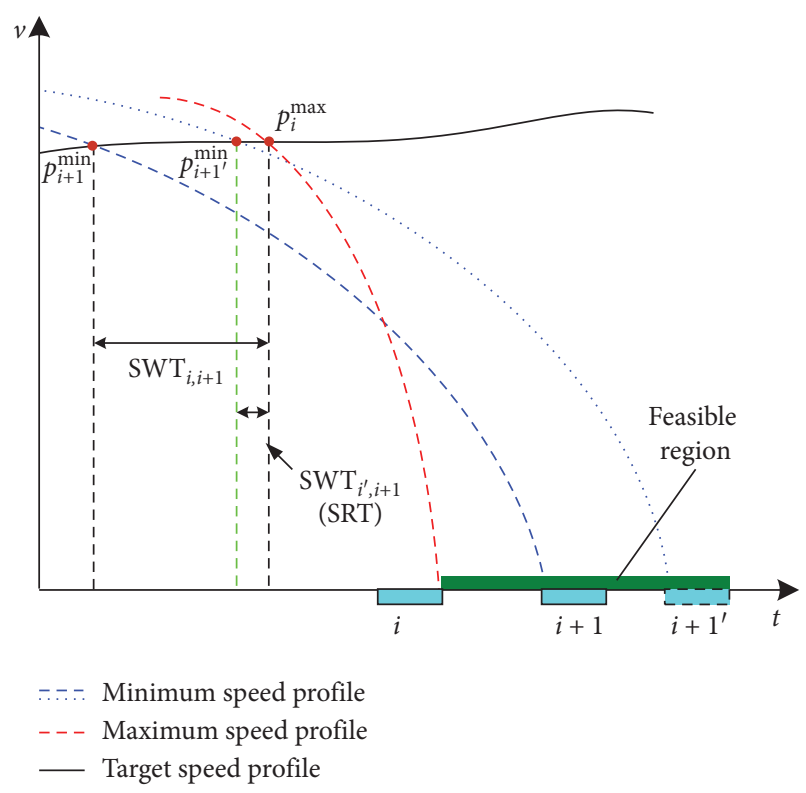

Figure 4: Characteristic of distance demand for ASAs.

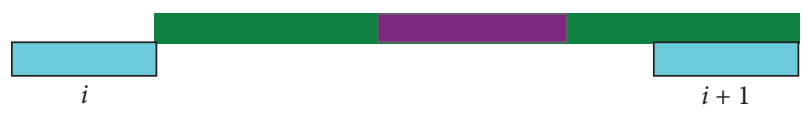

(a)

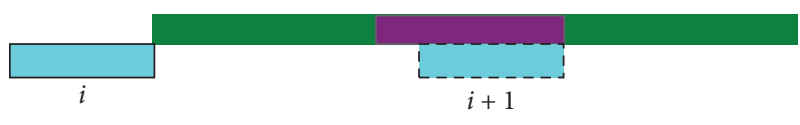

(b)

FIGURE 5: Strategy for tracking-oriented sections.

When a new ASA happens to be located at a demand restriction section, as shown in Figure 6(a), the result violates the constraint of Equation (7). According to the criterion that ASAs should be as far as possible from the origin station (Equation (1)), if the new ASA is shifted out of the demand restriction section to the right, the result will violate the constraint of Equation (3). Therefore, it can only be shifted to the left. When it happens to move out of the demand restriction section, the ASA is located at the farthest position from ASA $i$ or the origin station, as shown in Figure 6(b).

Based on the above analyses and the constraints of the model, we can take the origin station as the first reference ASA and determine ASAs step by step. The flow of the solution is shown in Figure 7.

At the same time, we can also start from the terminal station and determine the position of ASAs. The flow of the solution is shown in Figure 8.

\section{ASA Layout Method for Bidirectional Lines}

3.1. Problem Description. Compared with unidirectional lines, the ASA layout for bidirectional lines needs to consider the operation of the either direction. If the method in 


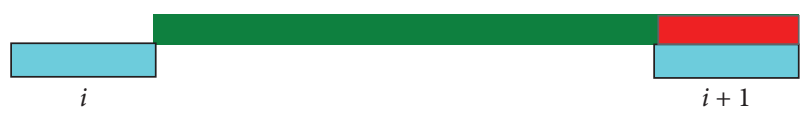

(a)

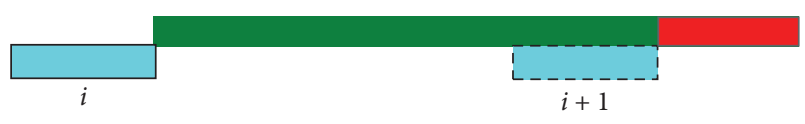

(b)

FIGURE 6: Strategy for demand restriction sections.

Figure 7 or Figure 8 is used for bidirectional lines, it is likely to result in excessive redundancy of ASAs. This paper further discusses and addresses this problem.

First, we describe the problem in an ideal condition, where the line condition is consistent, and the parameters of the maximum speed profile or the minimum speed profile for a train at any position are consistent. Then, if a maximum speed profile is acquired, the maximum speed profiles of other positions can be obtained by translation, and the same is true for minimum speed profiles.

As shown in Figure 9, there is a bidirectional HSM line. The direction from Station $O$ to Station $D$ is the positive direction, and the direction from Station $D$ to Station $O$ is the opposite direction. ASAs $8^{+}, 7^{+}, \ldots, 1^{+}$of the positive direction are obtained by using the method in Figure 8, and ASAs $1^{-}, 2^{-}, \ldots, 10^{-}$of the opposite direction are also obtained by the same method. The layout results show that the method in Figure 8 can obtain an effective optimal solution. However, the layout approach considering the directions separately causes the excessive redundancy of ASAs, as shown in Figure 9, which is not conducive to cost saving $[6,12,13]$.

In fact, there is no difference in the physical structure of ASAs for different directions. They are only marked as different directions from the perspective of train operation. Therefore, in an ASA layout process, some ASAs can be reasonably set as bidirectional ASAs (i.e., ASAs of the either direction) to reduce the overall number of ASAs.

3.2. Two-Way Coordination Layout Method. To address the problem above, this paper proposes an ASA two-way coordination layout algorithm (ASA-TWCLA). The method sets up the ASA of the two directions from Station $D$ step by step, as shown in Figures 7 and 8, and requires all ASAs to be as far as possible from Station $D$. The solution is introduced as follows.

As shown in Figure 10(a), there are two ASAs of the opposite direction (i.e., ASA $9^{-}$and ASA $10^{-}$) obtained based on Figure 7 and one ASA of the positive direction (i.e., ASA $6^{+}$) obtained based on Figure 8. For ASA $10^{-}$, ASA $9^{-}$is its reference ASA. That is to say, ASA $10^{-}$cannot be set farther away from Station $D$ on the basis of the method in Figure 7. For ASA $6^{+}$, according to Figure 4 and the stopping point stepping mode, it can satisfy the stopping point stepping requirement of the opposite direction (i.e., ASA 9 ${ }^{-}$ $\longrightarrow$ ASA $6^{+}$). Thus, in accordance with the criterion that ASAs should be as far as possible from the terminal station
(Equation (2)), ASA $6^{+}$can be set as a bidirectional ASA of the two directions (i.e., ASA 7 in Figure 10(b)) and the reference ASA for further determining the next ASA of the opposite direction. By doing this and combining with Figure 7, the new ASA of the opposite direction shown in Figure 10(b) can be obtained (i.e., ASA $10^{-}$). Certainly, ASA $10^{-}$in Figure $10(\mathrm{~b})$ is farther from Station $D$ than ASA $10^{-}$ in Figure 10(a).

Similarly, as shown in Figure 11(a), for the opposite direction, there are two ASAs (i.e., ASA $1^{-}$and ASA $2^{-}$) obtained based on the method in Figure 7. For the positive direction, there is one ASA (i.e., ASA $1^{+}$) obtained based on the method in Figure 8 , and the Station $D$ is its reference ASA. That is to say, the four ASAs are obtained considering the two directions separately, and they cannot be set farther away from Station $D$ according to the methods in Figures 7 and 8. However, according to Figure 4 and the stopping point stepping mode, ASA $1^{-}$and ASA $2^{-}$can satisfy the stopping point stepping requirement of the positive direction (i.e., ASA $1^{-} \longrightarrow$ Station $D$ or ASA $2^{-} \longrightarrow$ Station $D$ ), and ASA $2^{-}$is farther away from Station $D$ than ASA $1^{-}$. Thus, according to the criterion that ASAs should be as far as possible from the terminal station (Equation (2)), ASA 2can be set as a bidirectional ASA of the two directions (i.e., ASA 1 in Figure 11(b)) and the reference ASA for further determining the next ASA of the positive direction. By doing this and combining with Figure 8, the new ASA of the positive direction shown in Figure 11(b) can be obtained (i.e., ASA $1^{+}$). Then, ASA $1^{+}$in Figure 11(b) is farther from Station $D$ than ASA $1^{+}$in Figure 11(a).

According to Figures 10 and 11, when setting up a new ASA, by judging whether there are ASAs between it and its reference ASA, and taking corresponding actions, the goal that each new ASA is farthest from Station $D$ can be achieved. Taking the scenario in Figure 9 as an example, combining with Figures 7, 8, 10, and 11, the process of the ASA two-way coordination layout method is summarized as follows:

(i) Step 1. Take Station $D$ as the reference ASA and obtain the first ASA of the positive direction according to Figure 8 and the first ASA of the opposite direction according to Figure 7, respectively.

(ii) Step 2. Take the ASA farther from Station $D$ as the temporary ASA, and use the direction of the temporary ASA as the reference direction. At the same time, the ASAs in the direction opposite to the reference direction are further set until the position of the latest ASA is farther from Station $D$ than the temporary ASA.

(iii) Step 3. If the temporary ASA is located at a tracking-oriented section, take it as a bidirectional ASA and cancel the latest ASA in the direction opposite to the reference direction. Otherwise, cancel the latest ASA in the direction opposite to the reference direction and the temporary ASA and regard the ASA farthest from Station $D$ as a bidirectional ASA. 


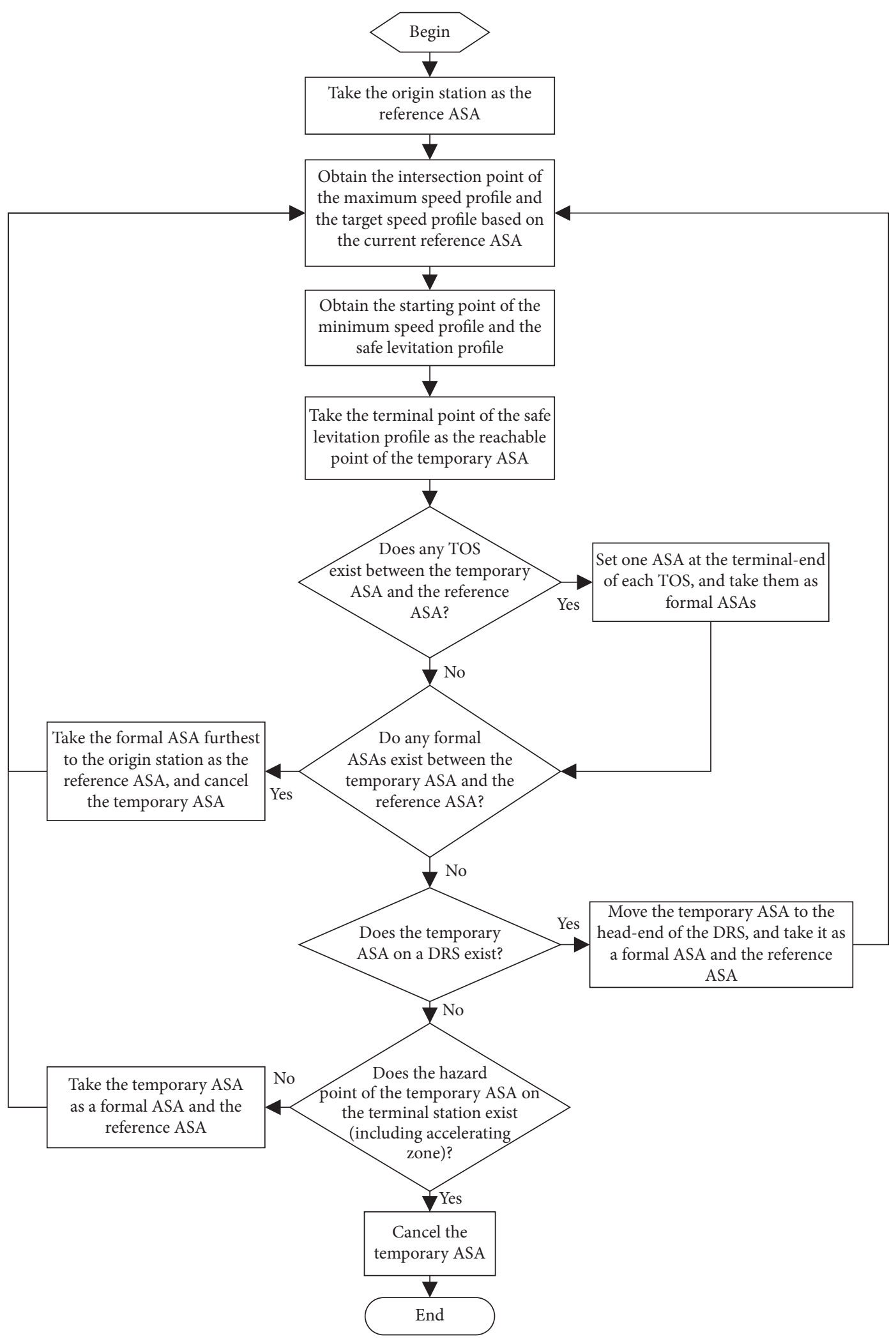

FIgURE 7: ASA layout starting from the origin station.

(iv) Step 4. Take the latest bidirectional ASA as the reference ASA, and obtain the next ASA of the positive direction and the next ASA of the opposite direction, respectively. If the new ASA of a direction is located at Station $O$ or its accelerating zone, the ASA is unnecessary, and the ASA layout of the 


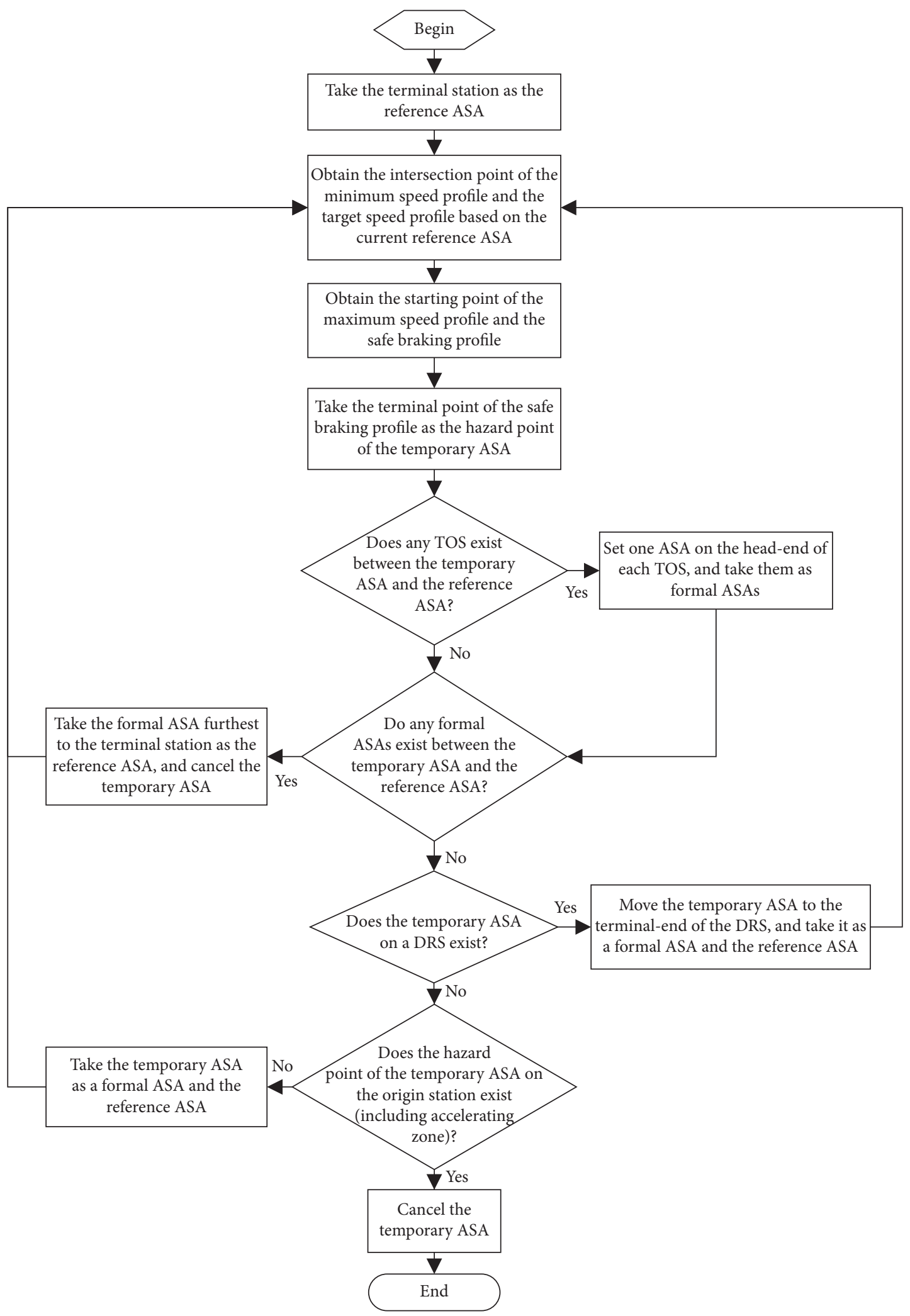

FIgURE 8: ASA layout starting from the terminal station.

direction is complete. After completing the ASA layout of the other direction according to the corresponding algorithm, the ASA layout of the line is end. Otherwise, go back to Step 2.
According to the above process, the ASA-TWCLA algorithm shown in Figure 12 is proposed. To distinguish between the ASAs of the positive direction and the ASAs of the opposite direction, the bidirectional ASAs are indicated 


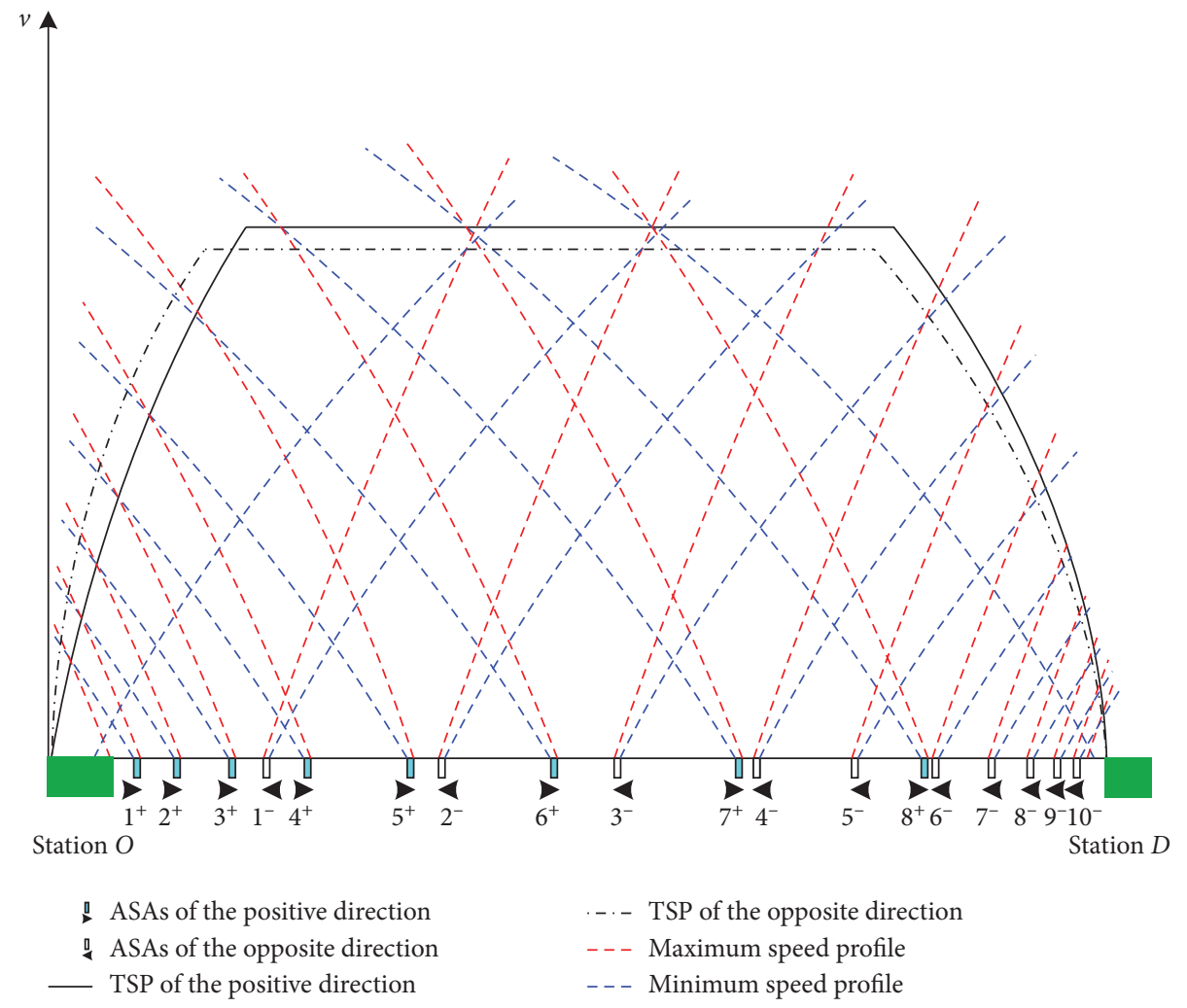

FIGURE 9: ASA layout considering the two directions separately for the ideal condition.

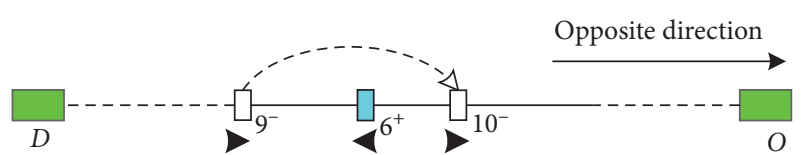

(a)

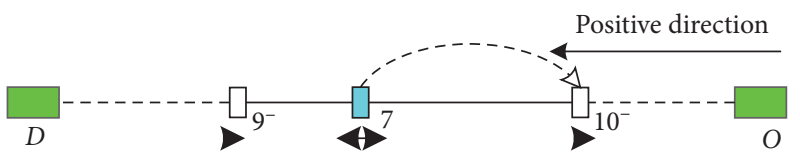

(b)

Figure 10: Bidirectional ASA determination based on an ASA of positive direction.

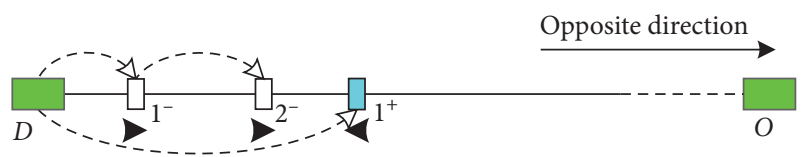

(a)

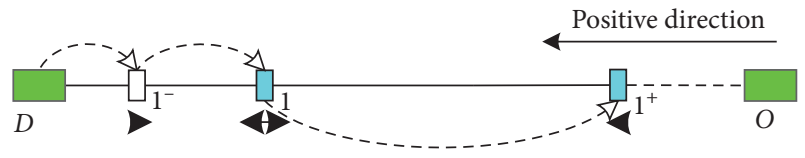

(b)

FIgURE 11: Bidirectional ASA determination based on an ASA of opposite direction. by $k$, ASAs of the positive direction that do not include bidirectional ASAs are indicated by $i$, and ASAs of the opposite direction that do not include bidirectional ASAs are indicated by $j$. At the same time, because the reachable point and the hazard point of an ASA are related to the train operation direction, in Figure 12, the mileage of the left margin for an ASA is recorded as $S_{i}$ or $S_{j}$, the length of an ASA is recorded as $l$, the mileage of the right margin for an ASA is recorded as $S_{i}+l$ or $S_{j}+l$, and the mileage of the right margin for Station $O$ is recorded as $S_{O}$.

For the scenario in Figure 9, Figure 13 shows the positions of ASAs obtained by the ASA-TWCLA algorithm. The result suggests that the method in Figure 12 can obtain an optimal solution. Meanwhile, there are relatively fewer ASAs than before $(14<18)$, including 3 ASAs of the position direction, 5 ASAs of the opposite direction, and 6 ASAs of the either direction. Compared with the basic layout methods, we believe that the algorithm can further reduce the number of ASAs in a same scenario.

\section{Numerical Experiment}

4.1. Experiment Design. In the experiment, a test line with the total length of $85730 \mathrm{~m}$ was employed. The terminal-end of power rails for the accelerating zone of Station $O$ was set to 2 $900 \mathrm{~m}$, and the head-end of power rails for the accelerating zone of Station $D$ was set to $82830 \mathrm{~m}$. Meanwhile, there were three propulsion decentralizations in the interstation section, and the boundary positions of them were $28385 \mathrm{~m}$ and $59335 \mathrm{~m}$. Table 1 shows the gradients of the line. Among them, 10 sections 


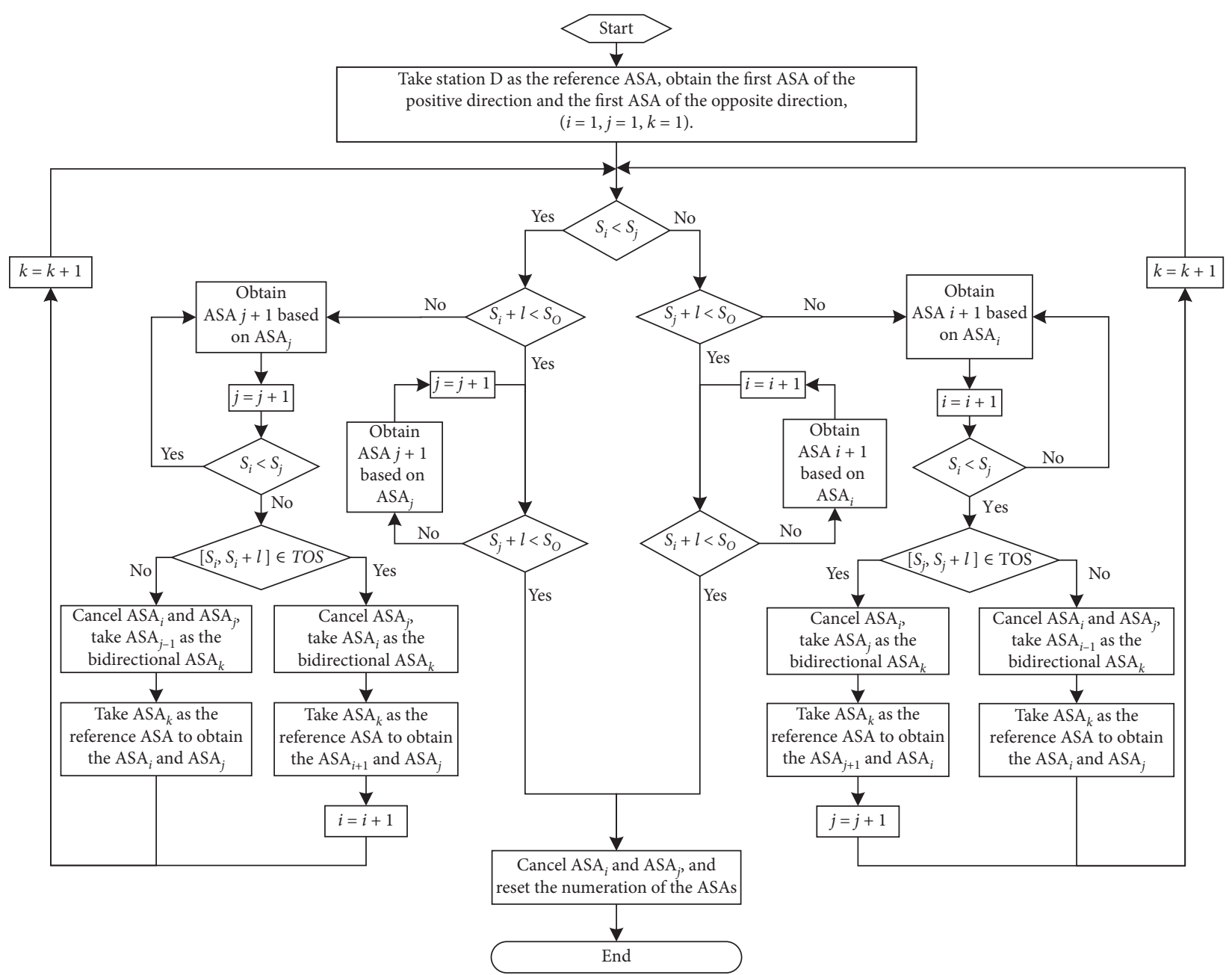

FIgURE 12: ASA two-way coordination layout algorithm.

(marked with “*”) have a gradient greater than $0.5 \%$, which are unsuitable for setting up ASAs according to the construction experience of the Shanghai Maglev Demonstration Line, and they are all demand restriction section. The train simulated had three vehicles, with a length of $79.5 \mathrm{~m}$ [2]. According to the construction experience of the Shanghai Maglev Demonstration Line, when the gradient is $0 \%$, the length of an ASA is set to the train length plus $180 \mathrm{~m}$, and when the gradient is $0.5 \%$, it takes the train length plus $250 \mathrm{~m}$. In view of the gradients of the test line where only a few sections have the gradient of $0 \%$, the length of the ASA in the study was set to $330 \mathrm{~m}$. Moreover, according to the study of Jiang et al. [13], to ensure the section tracking interval and the arrival tracking interval, combining with the protection requirement of a propulsion decentralization, 6 tracking-oriented sections were set up. The positions of them were [ 3400,3800$],[27485,27885],[28885,29285]$, [58 435, 58 835], [59 835, 60235$]$, and [81 930, 82 330].

At the same time, it was assumed that the reverse of the target speed profile for the position direction was the target speed profile for the opposite direction. The maximum speed of the maximum speed limit profile was set to $500 \mathrm{~km} / \mathrm{h}$, and the maximum speed of the target speed profile was $450 \mathrm{~km} / \mathrm{h}$. Meanwhile, the train dynamic model in Yu et al. [6] and the calculation method for the maximum speed profile and the minimum speed profile in Zhao et al. [7] were referenced, and the stepping redundancy time (SRT) was set to $10 \mathrm{~s}$.

For the numerical experiment, a simulation platform was built based on Visual Studio 2010 using $\mathrm{C}++$ Language. The direction that the mileage is increasing was regarded as the positive direction. The algorithms in Figures 7, 8, and 12 are analyzed and compared. In the simulations, the position of an ASA was rounded up to an integer.

4.2. Results. The ASA layout scheme obtained by the algorithm in Figure 7 for the opposite direction and the algorithm in Figure 8 for the positive direction is shown in Table 2 and Figure 14. There are 10 ASAs of the positive direction and 9 ASAs of the opposite direction, and there is no ASA at demand restriction sections. Among them, ASA 10 in Table 2 is the ASA obtained after an offset for the unsuitable section (i.e., Section 19 in Table 1) according to the approach in Figure 6, and ASAs 1, 7, 9, 13, 14, and 19 are at the tracking-oriented sections. The results further show that the method in Figures 7 and 8 can be used to assist the layout of ASAs for unidirectional HSM lines. 


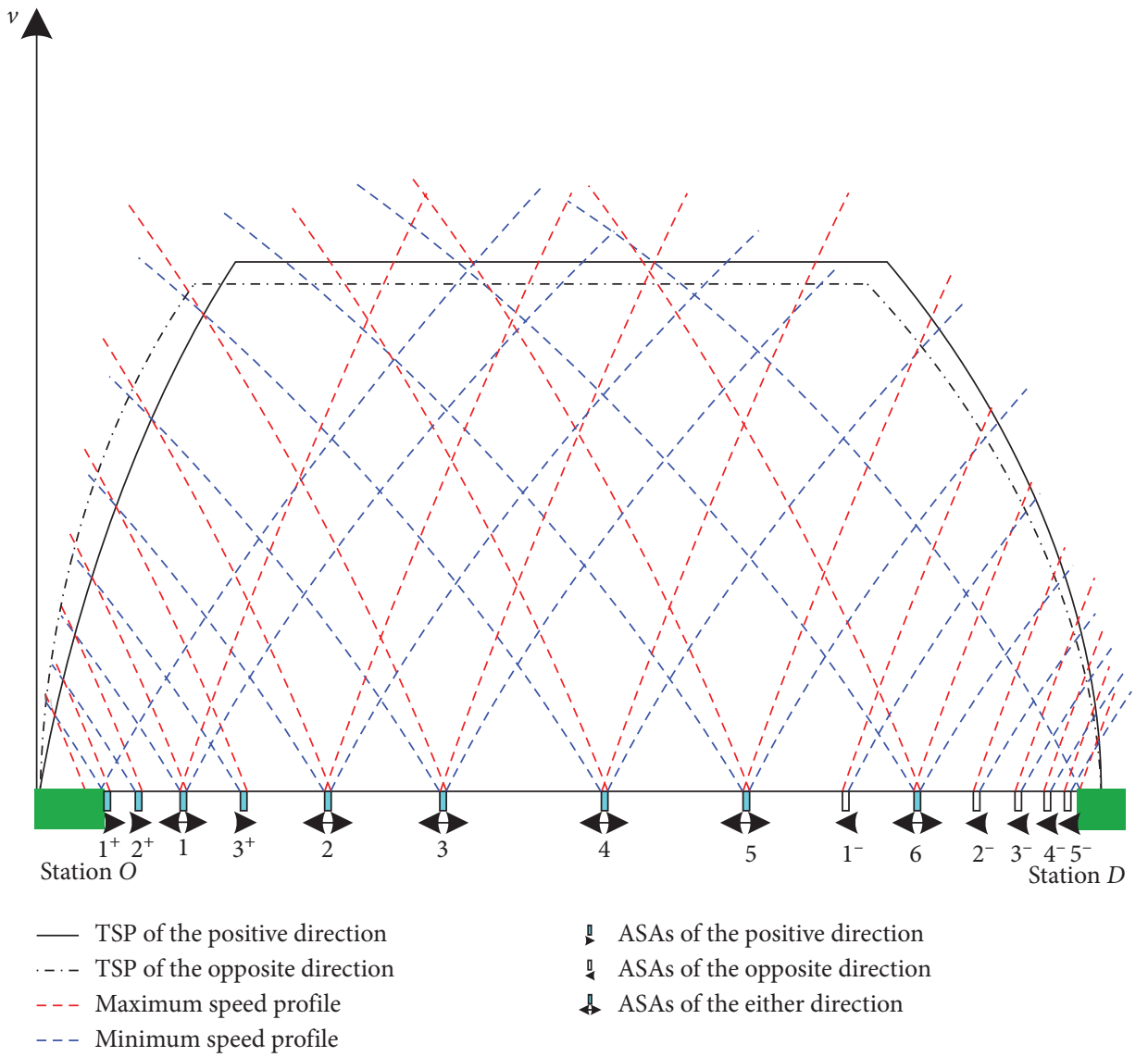

FIGURE 13: ASA layout considering the two directions coordinately for the ideal condition.

TABle 1: Basic data of the test line.

\begin{tabular}{|c|c|c|c|c|c|c|c|}
\hline No. & Origin position $(\mathrm{m})$ & Terminal position $(\mathrm{m})$ & Gradien (\%) & No. & Origin position $(\mathrm{m})$ & Terminal position $(\mathrm{m})$ & Gradient (\%) \\
\hline 1 & 0 & 2980.52 & 0.0000 & 21 & 37403.49 & 39283.61 & -0.0532 \\
\hline 2 & 2980.52 & 3280.52 & 0.3000 & 22 & 39283.61 & 40630.22 & -0.2970 \\
\hline 3 & 3280.52 & 4280.52 & -0.2000 & 23 & 40630.22 & 50221.55 & 0.0000 \\
\hline 4 & 4280.52 & 5080.52 & 0.0000 & 24 & 50221.55 & 53783.97 & 0.1825 \\
\hline $5^{*}$ & 5080.52 & 5350.82 & -0.5019 & $25^{*}$ & 53783.97 & 56950.00 & -0.8885 \\
\hline $6^{*}$ & 5350.82 & 5610.52 & 1.2000 & $26^{*}$ & 56950.00 & 57880.00 & 3.1226 \\
\hline 7 & 5610.52 & 6280.52 & -0.2500 & 27 & 57880.00 & 60831.31 & 0.0000 \\
\hline 8 & 6280.52 & 8185.52 & 0.0000 & 28 & 60831.31 & 62656.31 & -0.5000 \\
\hline 9 & 8185.52 & 9130.52 & 0.5000 & 29 & 62656.31 & 65246.31 & 0.0000 \\
\hline 10 & 9130.52 & 10926.23 & -0.2500 & $30^{*}$ & 65246.31 & 66000.00 & 0.8127 \\
\hline $11^{*}$ & 10926.23 & 12700.00 & 0.5533 & 31 & 66000.00 & 70451.31 & 0.2460 \\
\hline 12 & 12700.00 & 13520.00 & -0.3659 & $32^{*}$ & 70451.31 & 72000.00 & -1.0654 \\
\hline 13 & 13520.00 & 18630.22 & 0.0000 & 33 & 72000.00 & 75636.31 & 0.0000 \\
\hline 14 & 18630.22 & 20339.68 & 0.2164 & $34^{*}$ & 75636.31 & 76336.31 & 0.8000 \\
\hline 15 & 20339.68 & 22952.09 & -0.2182 & 35 & 76336.31 & 77896.31 & 0.3000 \\
\hline 16 & 22952.09 & 26599.56 & 0.0000 & $36^{*}$ & 77896.31 & 78796.31 & -1.2000 \\
\hline 17 & 26599.56 & 27285.2 & 0.4375 & 37 & 78796.31 & 80146.31 & 0.0000 \\
\hline 18 & 27285.20 & 29483.98 & -0.1364 & 38 & 80146.31 & 81286.31 & 0.4000 \\
\hline $19^{*}$ & 29483.98 & 30439.78 & 0.6277 & 39 & 81286.31 & 82336.31 & -0.4000 \\
\hline 20 & 30439.78 & 37403.49 & -0.0144 & 40 & 82336.31 & 85730.00 & 0.0000 \\
\hline
\end{tabular}

${ }^{*}$ Demand restriction sections.

As shown in Figure 14, the scheme has a phenomenon similar to that in Figure 9. For the fact that the algorithms in Figures 7 and 8 cannot fully optimize ASAs while considering the bidirectional operation, the ASA-TWCLA algorithm was used for the test line. The result obtained by the algorithm is shown in Table 3 and Figure 15. There are 14 
TABLE 2: ASA positions considering the two directions separately.

\begin{tabular}{lccc}
\hline ASA & Origin position $(\mathrm{m})$ & Terminal position $(\mathrm{m})$ & Direction \\
\hline 1 & 3400 & 3730 & Opposite \\
2 & 3930 & 4260 & Positive \\
3 & 6130 & 6460 & Positive \\
4 & 10123 & 10453 & Positive \\
5 & 13523 & 13853 & Opposite \\
6 & 16775 & 17105 & Positive \\
7 & 27485 & 29030 & Positive \\
8 & 28700 & 29215 & Positive \\
9 & 28885 & 30870 \\
10 & 30540 & 43586 & Opposite \\
11 & 43256 & 44995 & Opposite \\
12 & 44665 & 58765 & Positive \\
13 & 58435 & 60165 & Opposite \\
14 & 59835 & 66601 & Positive \\
15 & 66271 & 70220 & Opposite \\
16 & 69890 & 77499 & Positive \\
17 & 77169 & 81283 & Opposite \\
19 & 80953 & 82260 & Opposite \\
\hline
\end{tabular}

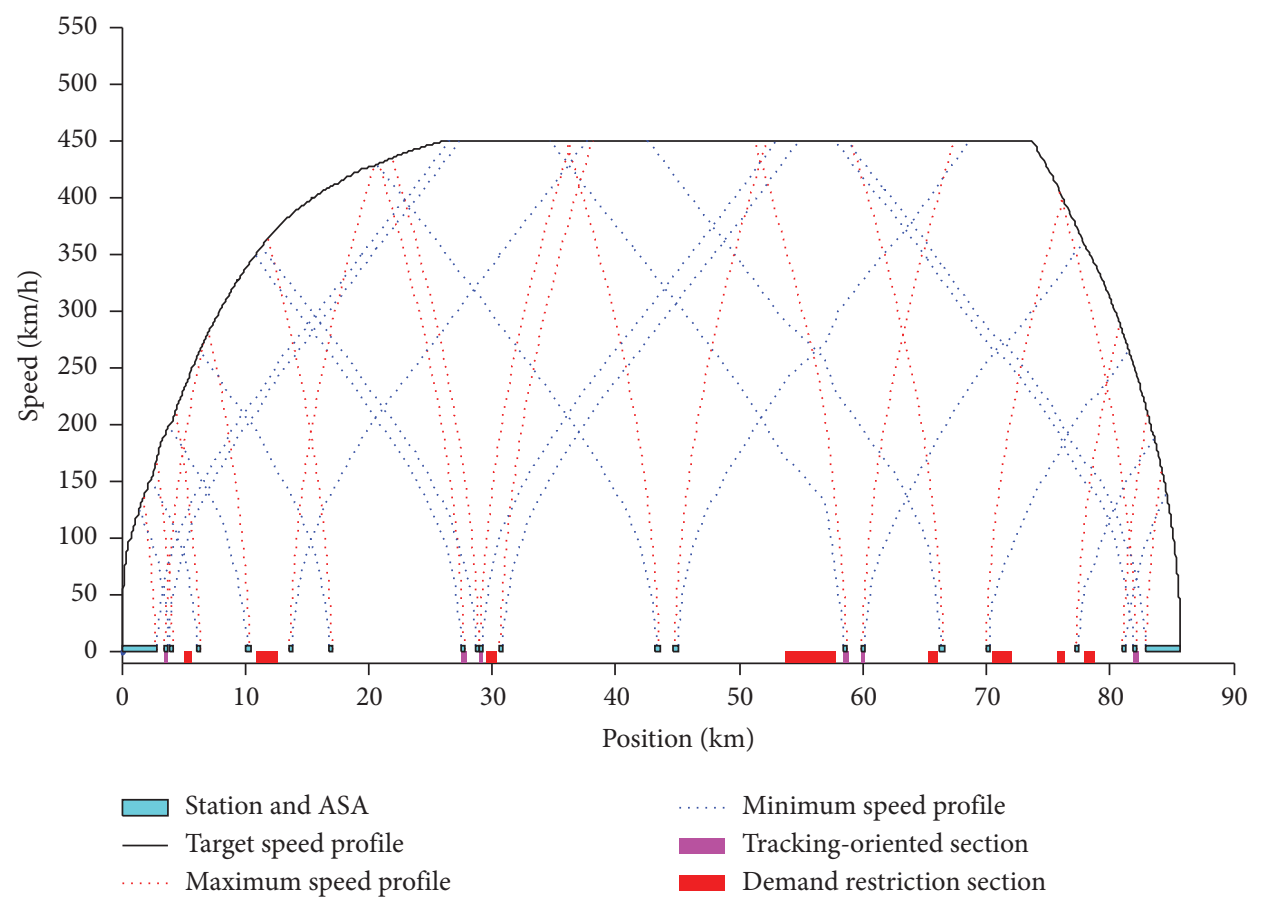

FIGURE 14: Layout scheme considering the two directions separately.

ASAs, including 4 ASAs of the positive direction, 3 ASAs of the opposite direction, and 7 ASAs of the either direction, and there is no ASA at demand restriction sections. Among them, ASA 11 in Table 2 is the ASA obtained after an offset for the unsuitable section (i.e., Section 30 in Table 1) according to the approach in Figure 6, and ASAs 1, 6, 7, 9, 10 and 14 are at the tracking-oriented sections. The results further demonstrate that the algorithm in Figure 12 can be applied to the ASA layout of bidirectional HSM lines.
4.3. Discussion. For the same condition, different ASA layout methods show different results, as shown in Table 4. The ASA two-way coordination layout algorithm (ASATWCLA) can reduce the total number of ASAs to a greater extent $(26.316 \%)$, and the construction cost of the line will also drop. At the same time, the optimized ASA layout scheme has increased the number of stopping points, but the result has no substantial impact on the design cost of an operation control system and the safety of train operation. 
TABLE 3: ASA positions considering the two directions coordinately.

\begin{tabular}{lccc}
\hline ASA & Origin position $(\mathrm{m})$ & Terminal position $(\mathrm{m})$ & Direction \\
\hline 1 & 3400 & 3730 & Opposite \\
2 & 3930 & 4260 & Positive \\
3 & 6130 & 6460 & Positive \\
4 & 10123 & 10453 & Positive \\
5 & 16775 & 17105 & Bidirectional \\
6 & 27485 & 27815 & Positive \\
7 & 28885 & 29215 & Bidirectional \\
8 & 43256 & 43586 & Bidirectional \\
9 & 58435 & 58765 & Bidirectional \\
10 & 59835 & 60165 & Bidirectional \\
11 & 66100 & 66430 & Opposite \\
12 & 74503 & 74833 & Bidirectional \\
13 & 79411 & 79741 & Opposite \\
14 & 81930 & 82260 & Bidirectional \\
\hline
\end{tabular}

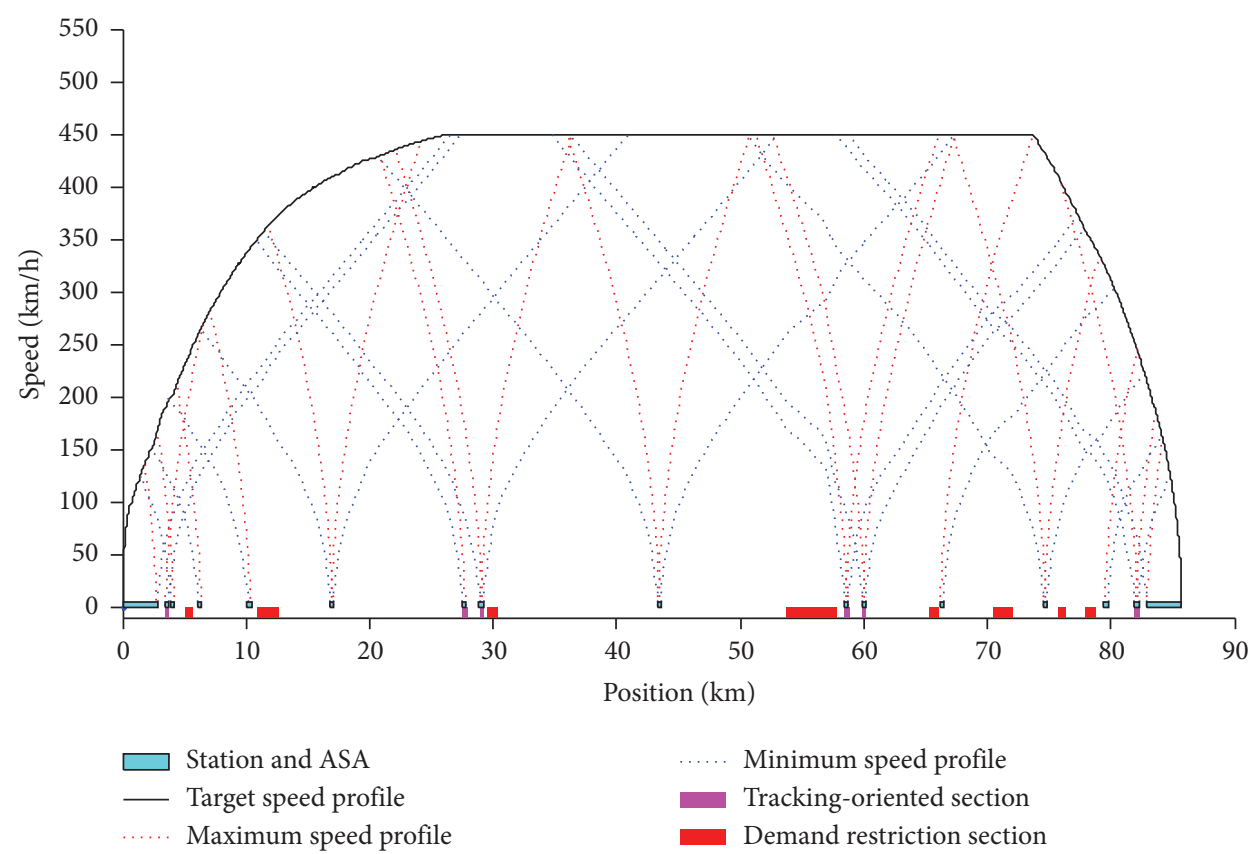

FIGURE 15: Layout scheme considering the two directions coordinately.

TABLE 4: Comparison of results.

\begin{tabular}{lcccccc}
\hline & & \multicolumn{2}{c}{ Number of ASAs } & \multicolumn{2}{c}{ Number of stopping points } \\
Layout method & Positive & Opposite & Bidirectional & Total & $\begin{array}{c}\text { Operating saving } \\
\text { positively }\end{array}$ & $\begin{array}{c}\text { Operating } \\
\text { oppositely }\end{array}$ \\
\hline $\begin{array}{l}\text { Separate layout (Figures 7, 8, and } \\
\text { 14) }\end{array}$ & 10 & 9 & 0 & 19 & 11 & 10 \\
ASA-TWCLA (Figures 12 and 15) & 4 & 3 & 7 & 14 & 11 & 10 \\
\hline
\end{tabular}

During the operation of a maglev line, the target speed profile may be multiple. For example, there are two main target speed profiles for the Shanghai Maglev Demonstration Line, with the maximum speeds of $430 \mathrm{~km} / \mathrm{h}$ and $300 \mathrm{~km} / \mathrm{h}$. For normal high-speed maglev lines, once ASAs are set up, due to the working principle of the stopping point stepping mode, the adjustable region for train speed profiles is limited $[2,6,21]$. As a result, the applicability of the scheme in Figure 15 to the target speed profile with the maximum speeds lower than $450 \mathrm{~km} / \mathrm{h}$ (e.g., $300 \mathrm{~km} / \mathrm{h}$ ) needs to be further tested. It is worth noting that $\mathrm{Yu}$ et al. [6] proposed an ASA layout method for the unidirectional line with 


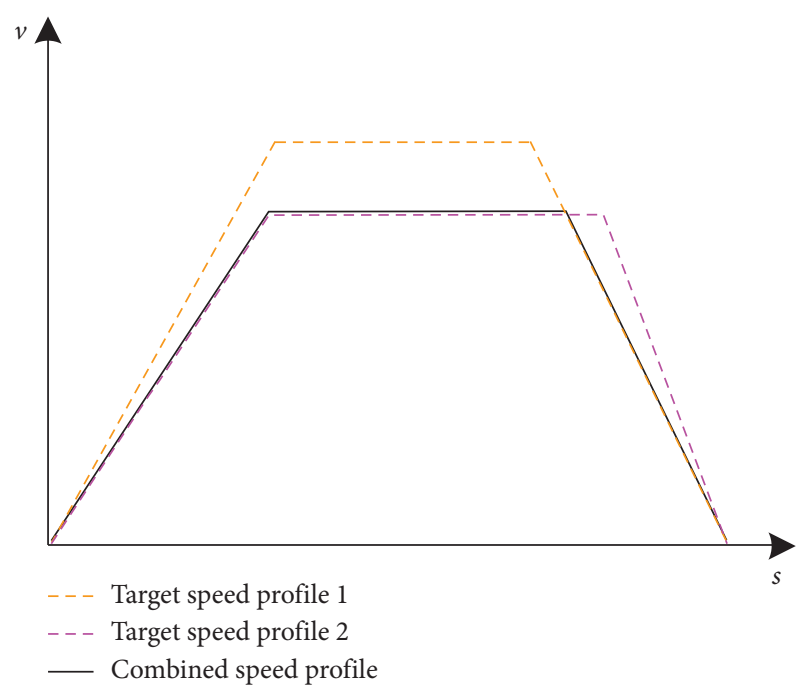

FIGURE 16: Abstraction of a combined velocity curve.

multitarget speed profiles. The method combines speed sections with the lowest speed among multiple target speed profiles into a combined speed profile as shown in Figure 16 and then uses the speed profile to set up ASAs. Because the method in this paper is applicable to any target speed profile, the abstract method of the combined speed profile can be considered for bidirectional lines with multitarget speed profiles, especially in the design stage of a line.

Meanwhile, the layout of ASAs is affected by gradients of the line. As discussed above, if an ASA obtained is located at an unsuitable section, we can offset the ASA towards its reference ASA. In the route selection process, in case the section unsuitable for ASAs is too long, the layout of ASAs should be considered. In addition, the length of an ASA can also be flexibly set, but whether the approach contributes to cost savings requires further study.

\section{Conclusion}

The study focuses on the bidirectional line of the normal highspeed maglev and analyzes the layout problem of auxiliary stopping areas (ASAs). The main contributions of the study are the ASA layout methods considering line conditions for unidirectional lines, the analysis of the ASA layout problem considering bidirectional operation, and the ASA two-way coordination layout algorithm (ASA-TWCLA). The results of the ideal condition and the numerical experiment show that the ASA-TWCLA algorithm can minimize the number of ASAs for a bidirectional line. For this paper, the influence of the ASA length on the economy of a layout scheme is ignored. The further study can consider the ASA layout problem with a more relaxation on the length of ASAs. Meanwhile, the application of the proposed method for the medium-speed maglev is also worth discussion.

\section{Data Availability}

The data used to support the findings of this study are available from the corresponding author upon request.

\section{Conflicts of Interest}

The authors declare that they have no conflicts of interest.

\section{Acknowledgments}

This work was supported by the National Key R\&D Program of China (grant no. 2016YFB1200602-02) and the Science Research Project of Shanghai Science and Technology Committee (grant no. 18DZ1205803).

\section{References}

[1] H. W. Lee, K. C. Kim, and J. Lee, "Review of maglev train technologies," IEEE Transactions on Magnetics, vol. 42, no. 7, pp. 1917-1925, 2006.

[2] X. Wu, Maglev Train, Shanghai Science and Technology Press, Shanghai, China, 2013.

[3] F. Xu, S. Luo, and Z. Deng, "Study on key technologies and whole speed range application of maglev rail transport," Journal of the China Railway Society, vol. 41, no. 3, pp. 40-49, 2019.

[4] P. Chu, Y. Yu, D. Dong, H. Lin, and J. Yuan, "NSGA-II-based parameter tuning method and $\mathrm{GM}(1,1)$-based development of fuzzy immune PID controller for automatic train operation system," Mathematical Problems in Engineering, vol. 2020, Article ID 3731749, 20 pages, 2020.

[5] C. Chen, J. Xu, G. Lin, Y. Sun, and F. Ni, "Model identification and nonlinear adaptive control of suspension system of highspeed maglev train," Vehicle System Dynamics, 2020.

[6] Y. Yu, X. Jiang, H. Lin, and H. Zhao, "Setting method of auxiliary stopping area for high-speed maglev based on protection speed," Journal of Tongji University (Natural Science), vol. 47, no. 9, pp. 1310-1316, 2019.

[7] H. Zhao, Y. Chen, Z. Liao, and Y. Yu, "Study of speed curve monitoring technology for high-speed maglev operation," in Proceedings of the 16th COTA International Conference of Transportation Professionals, pp. 1033-1045, Shanghai, China, July 2016.

[8] F. Chen and H. Xu, "Research on speed protection curve of German high-speed maglev transportation," China Science and Technology Information, vol. 18, no. 17, pp. 248-251, 2006.

[9] J. Liu and W. Wu, "Research on 2-D speed protection curve and its algorithm of high-speed maglev transportation," China Railway Science, vol. 23, no. 4, pp. 106-110, 2002.

[10] G. Yang and Z. Tang, "Study on safety speed protection of the high maglev train," Journal of Beijing Jiaotong University, vol. 31, no. 2, pp. 38-42, 2007.

[11] Q. Lai, J. Liu, L. Meng, X. Chai, Q. Wang, and Y. Xu, "Optimization of the auxiliary stopping area planning in the middle-to-high speed maglev," Transportation Systems and Technology, vol. 4, no. 2, pp. 141-151, 2018.

[12] J. Bian, Research on Properties of Maglev Train Operation Control System and Characteristics of Auxiliary Stopping Area, Zhejiang University, Hangzhou, China, 2006.

[13] X. Jiang, Y. Yu, Z. Deng, H. Lin, G. Chen, and H. Zou, “Setting optimization of auxiliary stopping area for high-speed maglev considering train tracking operation," Journal of Railway Science and Engineering, vol. 18, no. 2, pp. 325-333, 2021.

[14] I. A. Asnis, A. V. Dmitruk, and N. P. Osmolovskii, "Solution of the problem of the energetically optimal control of the motion of a train by the maximum principle," USSR Computational Mathematics and Mathematical Physics, vol. 25, no. 6, pp. 37-44, 1985. 
[15] J. Yang, J. Wu, B. Wang, and S. Lu, “Algorithm of target speed profile optimization of energy-efficient train operation based on improved heuristic genetic algorithm," Journal of the China Railway Society, vol. 41, no. 8, pp. 1-8, 2019.

[16] Y. Cao, Z.-C. Wang, F. Liu, P. Li, and G. Xie, "Bio-inspired speed curve optimization and sliding mode tracking control for subway trains," IEEE Transactions on Vehicular Technology, vol. 68, no. 7, pp. 6331-6342, 2019.

[17] L. Wang, L. Yang, Z. Gao, and Y. Huang, "Robust train speed trajectory optimization: a stochastic constrained shortest path approach," Frontiers of Engineering Management, vol. 4, no. 4, pp. 408-417, 2017.

[18] G. Yang, Study on High Speed Maglev Train Optimum Speed Curve and its Tracking Control, Beijing Jiaotong University, Beijing, China, 2007.

[19] X. Chai, J. Liu, Q. Lai, L. Meng, W. Liang, and Y. Xu, “Optimal energy-efficient trajectory planning for middle-speed maglev considering constraint of auxiliary stopping area," Journal of Central South University (Science and Technology), vol. 50, no. 6, pp. 1499-1506, 2019.

[20] Q. Lai, J. Liu, R. Zhao, Y. Luo, L. Meng, and Y. Xu, "Optimal trajectory planning for middle-to-high speed maglev based on dynamic programming with mutative spacing," Journal of Jilin University (Engineering and Technology Edition), vol. 49, no. 3, pp. 749-756, 2019.

[21] Q. Lai, J. Liu, A. Haghani, L. Meng, and Y. Wang, "Energyefficient speed profile optimization for medium-speed maglev trains," Transportation Research Part E, vol. 141, Article ID 102007, 2020.

[22] Y. Yang, N. Nan, W. Wei, W. Zhang, and Y. Liu, "A section decentralization operation strategy for medium-speed maglev train based on battery states," in Proceedings of the 37th Chinese Control Conference, pp. 7804-7809, Wuhan, China, 2018.

[23] Y. Yang, S. Ni, M. Wang, and G. Zhang, "Technical measures of controlling train headway on high-speed railway," $A d$ vances in Smart Vehicular Technology, Transportation, Communication and Applications, Springer, Cham, Switzerland, pp. 124-132, 2019.

[24] Y. Zhang, C. Tian, X. Jiang, and Y. Wang, "Calculation method for train headway of high speed railway," China Railway Science, vol. 34, no. 5, pp. 120-125, 2013.

[25] T. Sheng, Z. Tian, and S. Qu, "Study on the algorithm for train headway based on the simulation of operation by driver of high-speed railway," Advances in Smart Vehicular Technology, Transportation, Communication and Applications, Springer, Cham, Switzerland, pp. 3-10, 2019.

[26] C. Tian, S. Zhang, Y. Zhang, and X. Jiang, "Study on the train headway on automatic block sections of high speed railway," Journal of the China Railway Society, vol. 37, no. 10, pp. 1-6, 2015.

[27] J. Wang and J. Wang, "A new early warning method of train tracking interval based on CTC," IEEE Transactions on Intelligent Transportation Systems, pp. 1-7, 2017.

[28] S. He, R. Song, and T. Eastham, "Train headway models and carrying capacity of super-speed maglev system," JSME International Journal Series C, vol. 47, no. 2, pp. 518-522, 2004.

[29] W. Ding, J. Miao, Q. Lai, S. Huo, and Y. Xu, "Calculation method of train tracking interval for medium-speed maglev train with consideration of auxiliary-stop area," AIP Conference Proceedings, vol. 2073, no. 1, Article ID 020106, 2019.

[30] X. Xu, K. Li, and L. Yang, "Rescheduling subway trains by a discrete event model considering service balance," Applied Mathematical Modelling, vol. 40, no. 2, pp. 1446-1466, 2015.
[31] Q. Li, "Speed curve monitoring of shanghai maglev demonstration line," Urban Mass Transit, vol. 9, no. 2, pp. 47-49, 2006. 